> Les méthodes traditionnelles d'identification bactérienne par la détermination de quelques caractéristiques phénotypiques et l'appréciation de quelques propriétés physiologiques ont montré leurs limites, en particulier pour la détection des micro-organismes non ou difficilement cultivables. Elles ont conduit à la description d'une très faible partie de la diversité bactérienne existante et à la sous-estimation même de la richesse du monde vivant qui nous entoure. En médecine, plusieurs maladies - à l'évidence infectieuses - sont restées sans étiologie jusqu'à l'avènement des méthodes moléculaires fondées sur l'analyse phylogénétique des séquences d'ARNr l6S ou de gènes de protéines. Ces méthodes permettent, aujourd'hui, une détection et une reconnaissance fiable des pathogènes difficilement cultivables et la mise en œuvre de traitements appropriés. Le suivi des maladies infectieuses (légionelloses, choléra) dont les agents sont en état de «non-cultivabilité » dans l'environnement est également facilité. Au-delà des pathogènes, notre environnement contient des milliers de bactéries non ou difficilement cultivables, qui ont suscité le développement de nouvelles stratégies de culture et, plus récemment, de techniques dites de «méta- ou écogénomique ». Un aperçu de la diversité métabolique et du potentiel génétique tout à fait insoupçonnés de ces bactéries nous est promis dans les années à venir. <

\title{
La diversité \\ insoupçonnée \\ du monde \\ microbien
}

Catherine Dauga, Joël Doré, Abdelghani Sghir

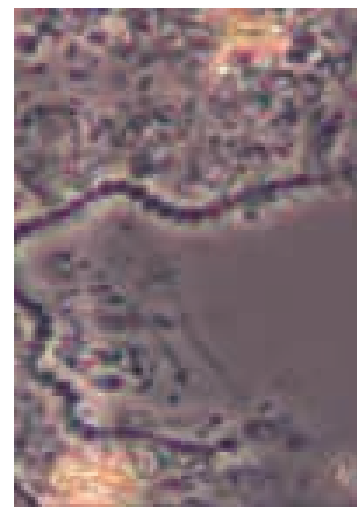

survie des populations humaines. Ils ont une importance cruciale dans la digestion et l'absorption des aliments que nous ingérons, et dans le développement de notre système immunitaire. Sans leur présence, la vie cesserait d'exister sur notre planète.

Les micro-organismes sont aussi la clé pour répondre à des questions aussi fondamentales que la nature et l'origine des premières cellules, le rôle de l'ADN, support de base de l'hérédité et du transfert de l'information du gène à la protéine. Par leur diversité, ils permettent des découvertes sur le métabolisme aussi bien que sur l'organisation des systèmes vivants. Parfois uniques et propres à ce monde invisible, ces nouvelles données sont aujourd'hui explorées grâce aux avancées technologiques de la biologie moléculaire et à l'utilisation de techniques et de stratégies de plus en plus sophistiquées.

\section{L’identification bactérienne, hier}

Les micro-organismes ont été les seuls êtres vivants sur notre planète pendant près de deux milliards d'années. Ils représentent, aujourd'hui, une biomasse totale de $10^{30}$ bactéries. Ils assurent les cycles biogéochimiques de l'oxygène, du carbone, de l'azote et d'autres éléments essentiels à la vie. Ils créent et assurent l'équilibre de l'atmosphère, la purification des eaux et la fertilisation des sols, ce qui les rend indispensables à la
Les premiers efforts d'identification bactérienne, nés des expériences de Louis Pasteur en 1870, se sont fondés sur les caractéristiques morphologiques, biochimiques et physiologiques. Les bactéries sont observées au microscope avec ou sans coloration. Leur forme, leur mobilité, les propriétés de leur paroi sont ainsi caractérisées. Elles sont aussi isolées sur des milieux de culture puis, en 
fonction des substrats qu'elles catabolisent, des éléments qu'elles respirent, leur profil métabolique est établi. Ces méthodes ont permis de différencier entre eux de nombreux micro-organismes et d'établir une classification phénotypique. Des schémas simplifiés, issus de ces descriptions, sont utilisés en pratique courante pour l'identification des bactéries d'intérêt médical.

La diversité bactérienne de l'environnement a été appréciée par ces mêmes méthodes phénotypiques jusque dans les années 1980. Cependant, appliquées à des habitats naturels complexes tels que le sol, les sédiments, l'eau douce et l'eau de mer, ces méthodes ont montré leurs limites. Dans leur habitat naturel, la plupart des micro-organismes existent sous forme non cultivable. Il s'agit soit de micro-organismes méconnus qui ne peuvent croître sur les milieux de culture disponibles dans les laboratoires, soit de micro-organismes connus (habituellement cultivables) dans un état de dormance difficilement réversible. L'avènement de la biologie moléculaire, dans les années 1990, va apporter de nouveaux moyens pour l'étude de ces organismes « non cultivables ». L'amplification enzymatique de I'ADN (PCR) permet, en effet, l'accès à la séquence des gènes bactériens sans étape de culture.

\section{L'approche moléculaire, aujourd'hui}

L'identification moléculaire des bactéries repose sur un constat: chaque bactérie possède un génome qui lui est propre. Les mutations s'accumulent progressivement au cours des générations et permettent l'évolution des gènes et des espèces. La séquence d'un gène résulte de l'héritage d'un gène ancêtre et de l'accumulation de mutations au cours du temps ${ }^{1}$. L'exploitation phylogénétique des différences observées entre les séquences des gènes bactériens ${ }^{2}$ permet de reconstituer les relations généalogiques entre les organismes.

Les méthodes phylogénétiques, au-delà de la simple appréciation qualitative ou quantitative des différences ou des similitudes, reconstituent l'histoire des séquences en fonction des processus évolutifs qu'elles ont subi. Parmi ces méthodes, les méthodes de distance transforment les différences observées entre les séquences en distances évolutives, tenant compte des événements substitutifs passés. Ces distances sont ensuite agglomérées dans un arbre phylogénétique, dont

${ }^{1}$ Ces mutations sont dues aux erreurs des polymérases qui surviennent pendant la réplication du génome lors de la division bactérienne. Elles résultent aussi des modifications plus ou moins spontanées des bases nucléiques, liées à leur instabilité chimique ou à l'instabilité de la liaison $\mathrm{N}$-glycosidique entre la base et le désoxyribose des nucléotides.

${ }^{2}$ Selon l'hypothèse où elles seraient représentatives de l'évolution de l'ensemble du génome. les longueurs de branches représentent les taux de mutations, et où les nœuds donnent la position probable des épisodes de spéciation. Les familles, les genres et les espèces bactériennes sont reconnus comme groupes d'organismes issus d'un même nœud, c'est-à-dire quand ils partagent un épisode de spéciation à un moment de leur histoire. L'avantage d'une classification phylogénétique par rapport à la classification phénotypique classique est de pouvoir reconstituer les relations entre tous les groupes bactériens, y compris ceux sans caractéristiques phénotypiques communes évidentes.

L'ARN ribosomique $16 S$ (ARNr 16S) a été choisi comme marqueur phylogénétique du fait de son universalité liée à son rôle clé dans la traduction de l'ARNm en protéines, de sa structure mosaïque incluant des régions conservées, variables et hypervariables, et de son abondance dans les cellules [1]. À ce jour, plus de 92000 séquences d'ARNr $16 \mathrm{~S}$ ou du gène qui code pour lui (ADNr l6S) sont à la disposition des scientifiques sur le réseau internet, dans des bases de donnés généralistes comme Genbank (http://www.ncbi.nlm.nih.gov/), ou des bases de données spécialisées comme le ribosomal database project (RDP) (http://rdp.cme.msu.edu) à I'Université du Michigan aux États-Unis ou the European ribosomal RNA database à l'Université de Gand en Belgique (http://www.psb.ugent.be/rRNA/index.html).

Aujourd'hui, n'importe quelle bactérie est identifiable par la position de sa séquence d'ADNr 16S dans un groupe représentatif d'une famille, d'un genre ou d'une espèce bactérienne au sein d'un arbre phylogénétique (Figure 1). Cette position phylogénétique est corrélée à la valeur de similitude des ARNr 16S: deux bactéries appartiennent à des espèces différentes si leurs ARNr 16S partagent moins de $97 \%$ de similitude [2]. Cependant, il semble raisonnable d'apprécier cette « limite » au cas par cas, car le taux de mutations observé dépend non seulement du temps écoulé entre les épisodes de spéciation, mais aussi de l'efficacité des mécanismes de réparation de I'ADN propres à chaque espèce.

L'identification bactérienne par séquençage de I'ARNr 165 est aujourd'hui complètement intégrée au processus de caractérisation des nouvelles bactéries ne correspondant à aucun profil phénotypique connu (Figure 2). De nouvelles espèces à caractère pathogène sont sans cesse découvertes: Mycobacterium heckeshornense, Leptotrichia trevisanii, Brevibacterium paucivorans, Laribacter hongkongensis, Corynebacterium tuberculostearicum..., pour n'en citer que quelquesunes.

Ces études ont également permis la reconnaissance de milliers de bactéries non cultivables dans les écosys- 
tèmes environnementaux et ont considérablement augmenté le nombre de lignées bactériennes. Nous sommes passés des 12 divisions (lignées ou phylums) décrites en 1987 par C. Woese [1] à 52 divisions en 2003 , dont 26 sans aucun micro-organisme cultivable [3-4]. Si la plupart des branches de l'arbre phylogénétique étaient initialement distinctes les unes des autres, l'abondance des séquences rend, aujourd'hui, leurs limites plus floues. La faible variabilité des séquences d'ARNr $16 S$ rend également confuse la limite des genres et des espèces bactériennes. De nouveaux marqueurs phylogénétiques: gyrB (gyrase) [5], rpoB (ARN polymérase) [6], hsp60 (heat-shock protein) [7]... ont été proposés en association à l'ARNr 165 pour la caractérisation de certaines espèces et genres bactériens cultivables. Ils devront être évalués pour la détection des bactéries non cultivables. Élaborer la classification naturelle des micro-organismes et augmenter la précision de l'identification moléculaire restent et resteront, dans les prochaines années, un des défis de la bactériologie.

\section{L'identification moléculaire et ses applications médicales}

Dans le domaine médical, l'identification bactérienne par séquençage de I'ARNr 165 a d'abord permis de découvrir l'étiologie de plusieurs maladies à agents non cultivables. Ainsi, Bartonella henselae [8], puis B. clarridgeiae, ont été identifiées comme agents responsables de la maladie des griffes du chat, une lymphadénite subaiguë parfois suppurée succédant à une griffure de chat. Bartonella henselae et $B$. quintana ont été incriminées dans l'angiomatose bacillaire, une infection dermatologique observée surtout chez les sujets présentant un syndrome d'immunodéficience acquise, dont les lésions peuvent ressembler à celles du sarcome de Kaposi, mais qui sont sensibles à l'érythromycine [9]. La maladie de Whipple, une lipodystrophie intestinale responsable d'un syndrome de malabsorption accompagné d'arthralgies et de fièvre, est formellement rattachée à une infection par Tropheryma whippelii [10] favorisée par un défaut d'immunité cellulaire, et son trai- tement fait appel à des antibiotiques. Aujourd'hui, la culture de ces agents pathogènes, bien que fastidieuse, est réalisable [11, 12], et de nombreux systèmes $P C R$ diagnostiques sont également disponibles $[13,14]$.

L'identification bactérienne par séquençage de I'ARNr l6S a pallié également les difficultés rencontrées pour l'identification de pathogènes à croissance très lente ne répondant pas à des tests bactériologiques conventionnels. Mycobacterium genavense, bactérie à croissance difficile, confondue avec $M$. fortuitum sur la base des profils d'acides gras, est identifiée par la spécificité de sa séquence d'ARNr 16S. La base de donnée RIDOM (http://www.ridom-rdna.de/) est spécialement dédiée à l'identification des mycobactéries.

Des applications quotidiennes ont aussi été développées pour l'identification de pathogènes rendus non cultivables au sein de tissus infectés, en raison de conditions locales particulières ou de l'usage préalable d'antibiotiques. Ainsi, Inquilinus limosus est une nouvelle espèce isolée de prélèvements de mucoviscidose [15]. Des germes de la cavité oropharyngée, comme Dialister sp, ont pu être identifiés comme agents responsables d'abcès cérébraux [16]. Dans ces quelques exemples, la responsabilité de l'agent pathogène dans l'infection n'est plus démontrée par l'ensemble des éléments du postulat de Koch ${ }^{3}$. Cette preuve est apportée essentiellement par la détection répétée du germe dans les cas cliniques où il est incriminé.

Dans le domaine médical, l'identification par séquençage de l'ARNr 165 présente malheureusement des limites liées au mode de définition

${ }^{3}$ Le troisième élément du postulat de Koch, qui nécessite de récréer la maladie dans un autre organisme en injectant l'agent pathogène préalablement isolé et caractérisé, n'est pas satisfait.

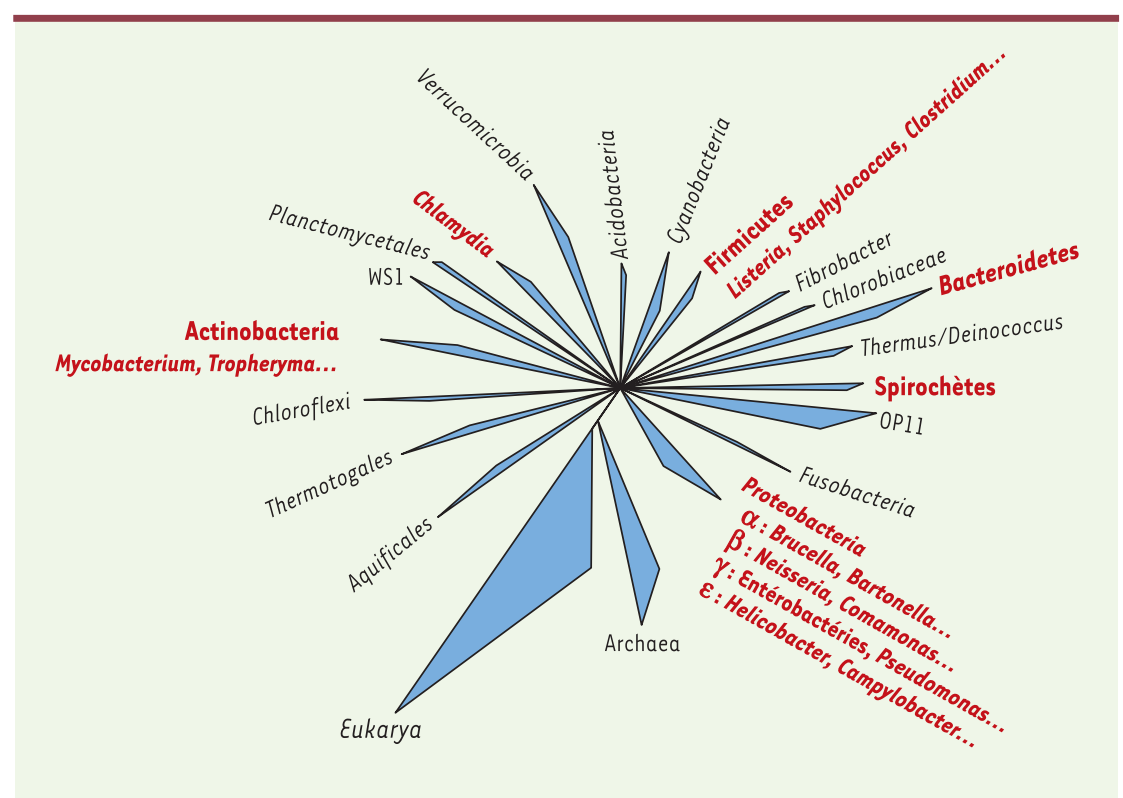

Figure 1. Positionnement des principales bactéries d'intérêt médical (en rouge) et de l'environnement parmi 18 divisions phylogénétiques d'eubactéries. À côté des divisions représentées par au moins un représentant cultivable, d'autres divisions sont nommées à partir des séquences d'inventaires moléculaires (OPll et WS1) et ne sont représentées, à ce jour, par aucun microorganisme cultivable (d'après [43]). 
de l'espèce bactérienne. Ainsi, certains pathogènes, $\varepsilon$. coli et Shigella spp, Bacillus cereus et $B$. anthracis ou Yersinia pseudotuberculosis et $y$. pestis, sont classés dans des espèces différentes en raison de leur virulence distincte, mais ils appartiennent à des espèces génomiques identiques et ne sont pas distingués par leur séquence d'ARNr 16S. Les espèces du genre Brucella sont aussi difficilement identifiées en raison du trop faible niveau de divergence de leur ARN ribosomique. Sur I'ARNr 16S, une mutation survient en moyenne tous les un à deux millions d'années, et des événements évolutifs récents ne seront pas toujours détectables. Il est alors nécessaire d'utiliser d'autres marqueurs génétiques pour permettre la discrimination de ces pathogènes.

\section{Exploration microbiologique et détection des pathogènes dans l'environnement}

Les agents pathogènes dans leur contexte environnemental L'analyse des ARNr 165 permet également l'identification des niches permettant la survie des pathogènes dans l'environnement.

\section{Le choléra}

Le choléra est une maladie diarrhéique due à des souches appartenant aux sérogroupes 01 et 0139 de Vibrio cholerae, un bacille isolé en 1883 par Koch en Égypte. L'homme joue à la fois un rôle de milieu de culture et de vecteur pour le vibrion cholérique. II se contamine par voie digestive: eaux de boisson, aliments, fruits et légumes, poissons, crustacés, fruits de mer. Les selles diarrhéiques, libérées en grande quantité, sont responsables de la propagation des bacilles dans l'environnement et de la transmission orofécale. La période d'incubation favorise le transport des vibrions sur de plus ou moins longues distances. Des études récentes d'identification moléculaire ont permis la détection de souches non cultivables d'espèces du genre Vibrio dans le milieu marin [17]. Une association entre V. cholerae et le zooplancton, en particulier avec des copépodes, a été mise en évidence [18].

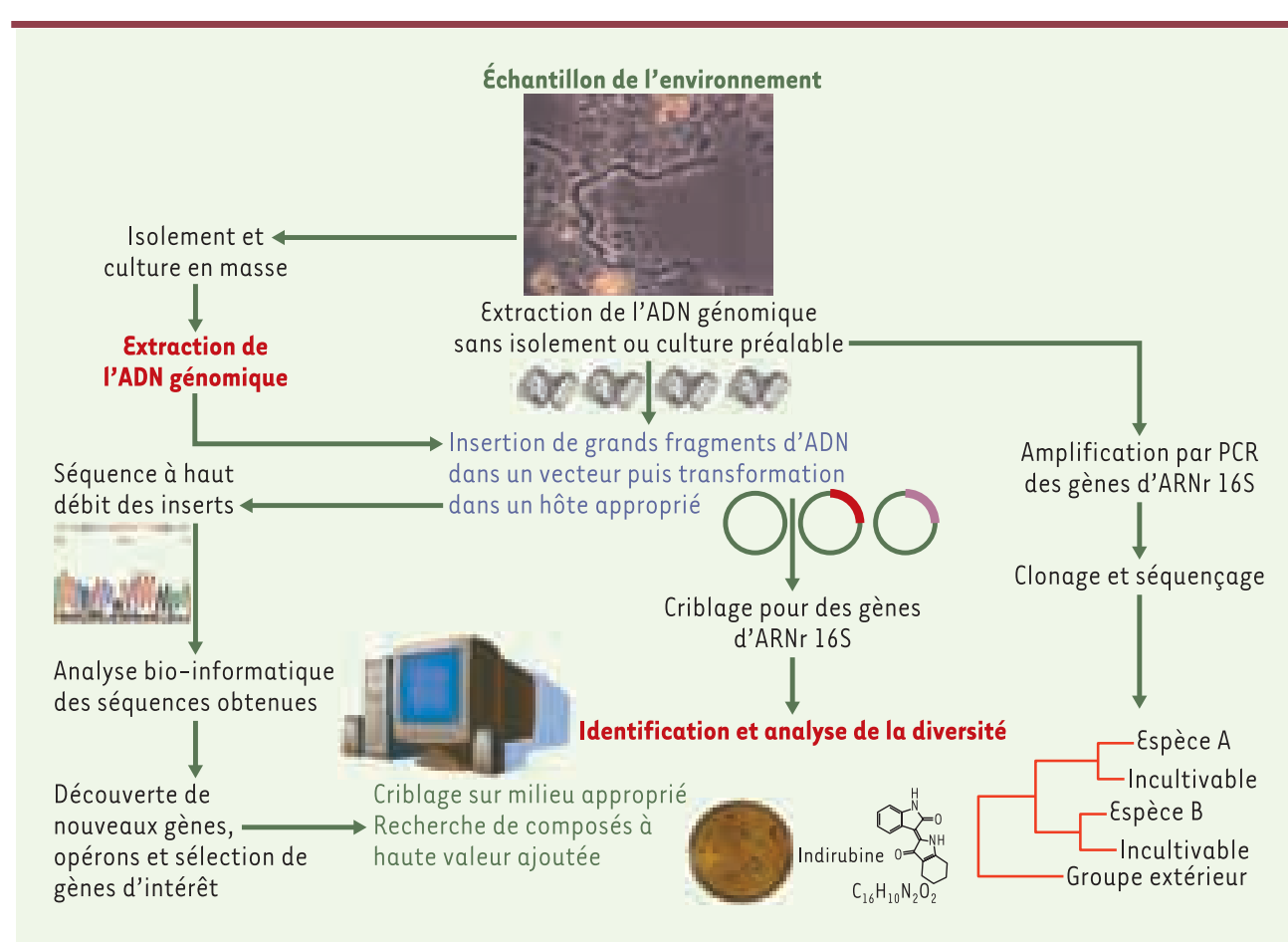

Figure 2. Stratégies d'identification bactérienne et d'exploration métagénomique par clonage et séquençage de grands fragments d'ADN génomique. À partir d'un échantillon complexe de l'environnement ou d'un enrichissement obtenu par culture, I'ADN génomique total est extrait, des fragments de haut poids moléculaire sont insérés dans des vecteurs BAC (bacterial artificial chromosome) ou fosmides, puis transformés dans $\varepsilon$. coli. Des banques de clones contenant les inserts de haut poids moléculaire sont ainsi construites. Le séquençage à haut débit couplé à l'analyse bio-informatique des séquences obtenues permet d'étudier des milliers de gènes et d'opérons différents. Les clones contenant les gènes d'intérêt sont criblés sur milieux appropriés pour la recherche de nouvelles activités. Le criblage de ces banques pour les gènes de l'ARNr 16S permet d'identifier l'organisme responsable des activités à haute valeur ajoutée. Ainsi sont reliées les deux approches, phylogénétique et génomique.

\section{La légionellose}

Les légionelles, responsables des maladies connues sous le nom de légionelloses, sont avant tout des bactéries de I'environnement, retrouvées dans les eaux douces et les lacs, ainsi que dans les sources chaudes, la terre et les composts. Elles vivent à l'état libre dans l'environnement. Le support environnemental de la multiplication intracellulaire des légionelles est constitué par les protozoaires. Le mauvais entretien des réseaux d'eau facilite le développement des Legionella, notamment au niveau des biofilms. Dans plus de $90 \%$ des cas, l'infection humaine est due à Legionella pneumophila. La maladie apparaît après inhalation de gouttelettes d'eau (aérosols) contaminées par des Legionella. $\varepsilon$ n raison de la culture 
délicate des légionelles ${ }^{4}$, les méthodes moléculaires se sont révélées très utiles et supérieures aux techniques bactériologiques classiques par leur sensibilité, leur rapidité et leur spécificité. Des protocoles utilisant la PCR et des amorces ciblant des régions spécifiques de l'ARNr 16S, I'ARNr 5S, rpoB (RNA polymerase gene) ou encore, le gène mip (macrophage infectivity potentiator) pour l'identification spécifique des légionelles, ont été décrits [19-24]. Dans certains cas, ils ont permis la détection de légionelles dans des échantillons dont les cultures sont restées négatives [25]. Dans d'autres cas, ils ont permis la détection de nouvelles espèces de légionelles à partir des filtres de sable utilisés pour la purification de l'eau [26]. En novembre 2003, 71 cas de maladie du légionnaire ont été rapportés dans le nord de la France [27]. Les techniques moléculaires ont permis de confirmer la présence des mêmes souches de Legionella dans 14 échantillons cliniques et dans les tours aéroréfrigérées du site pétrochimique Noroxo.

\section{Nouvelles lignées bactériennes potentiellement pathogènes présentes dans l'environnement}

Les études moléculaires fondées sur les séquences du gène de I'ARNr 16S des bactéries endosymbiotiques des amibes (Acanthamoeba sp), présentes dans le sol et les milieux aquatiques, ont montré l'existence de plusieurs lignées phylogénétiques nouvelles et potentiellement pathogènes. Certaines de ces lignées sont très proches de bactéries intracellulaires obligatoires appartenant au groupe des $\alpha$-protéobactéries, comme les Rickettsiae [28], tandis que d'autres sont affiliées à des Bacteroidetes [29], ou proches des Chlamydiae [30]. Dans cette dernière lignée, deux nouveaux genres, Parachlamydia et Neochlamydia, ont été décrits [31, 32].

\section{Perspectives}

Ainsi, une grande diversité bactérienne existe, bien plus grande que ne pouvait le laisser supposer notre vision étroite et biaisée d'un monde bactérien cultivable, et beaucoup reste encore à découvrir. II suffit, sans doute, d'investir du temps et des moyens pour isoler et caractériser un plus grand nombre de bactéries et mettre à jour cette diversité cachée.

\section{Cultiver le non cultivable :}

\section{un défi pour les microbiologistes d'aujourd'hui}

La proportion de bactéries cultivables dans les milieux naturels est très faible. Seulement $1 \%$ des bactéries du sol sont cultivables. Les raisons de cette «non cultivabilité » sont multiples. Il peut s'agir d'un état physiologique viable non cultivable ou de l'inadéquation des milieux de culture conventionnels à la physiologie de la bactérie. Enfin, les bactéries vivant en communautés, des interactions majeures entre

${ }^{4}$ Confection de milieux spéciaux et 3 à 5 jours de culture pour observer des colonies visibles. micro-organismes existent, qui résultent de l'existence même de la biodiversité de l'environnement naturel. Ce genre d'interactions disparaît dès que nous essayons de les cultiver in vitro et, avec elles, une diversité importante échappe à notre connaissance.

Plus l'effort est mis sur la recherche de stratégies innovantes, mieux on arrive à caractériser les micro-organismes de l'environnement. Le groupe de Karl Stetter [33] a été le premier à réussir à isoler un micro-organisme «non cultivable » en utilisant des techniques de micromanipulation combinées à l'utilisation de sondes d'hybridation moléculaires. Depuis, de nombreux microorganismes connus d'après les inventaires moléculaires du bactérioplancton marin ont été isolés. C'est le cas des micro-organismes SARll affiliés au groupe des $\alpha$-protéobactéries, des $\beta$-protéobactéries 0M43, des $\gamma$-protéobactéries 0M60/0M241 [34], ainsi que de certains représentants de nouvelles lignées phylogénétiques de micro-organismes telluriques et de micro-organismes de la flore digestive [35]. Des bactéries appartenant aux divisions des Actinobacteria, des Acidobacteria, des Verrucomicrobia ont aussi été récemment isolées et caractérisées [36, 37].

Les techniques de cultures proposées pour les micro-organismes marins utilisent des chambres de diffusion constituées de membranes permettant l'échange des molécules, mais pas celui des micro-organismes. La chambre elle-même est placée dans un aquarium simulant l'environnement naturel des bactéries [38]. Le nombre de micro-organismes récupérés à partir d'un échantillon marin cultivé de cette façon est 300 fois supérieur à celui observé après culture sur une boîte de Pétri classique. Ainsi, l'exploration de la diversité microbienne ne peut s'affranchir de la connaissance de la physiologie des microorganismes.

\section{Acquérir de nouvelles connaissances grâce à la génomique}

Les années 2000 nous offrent, avec le développement des grands centres de séquençage et de la génomique, l'accès au génome des bactéries cultivables ainsi qu'à ceux des bactéries de l'environnement: c'est «l'écogénomique ou la métagénomique ». L'exploration du métagénome ${ }^{5}$, c'est-à-dire de l'ensemble des génomes des micro-organismes, représente une nouvelle stratégie conduisant à un inventaire complet des gènes présents dans un environnement naturel. Une approche systématique de séquençage aléatoire à haut débit de milliers de clones de banques métagénomiques donne une bonne idée de leur contenu génétique et de l'organisation de leurs génomes (Figure 2).

\footnotetext{
${ }^{5}$ Cette méthode consiste, après extraction de l'ADN de l'environnement, à cloner dans des vecteurs connus sous le nom de BAC (bacterial artificial chromosome) chez $\varepsilon$. coli, et à séquencer de grands fragments d'ADN de taille comprise entre 50 et $150 \mathrm{~kb}$.
} 
En appliquant une variante de cette méthode à des populations microbiennes de la mer de Sargasse, J.C. Venter et al. ont produit des données considérables concernant le contenu génique de la microflore marine. Un million deux cent mille gènes, inconnus jusqu'alors, ont été mis en évidence. Ces gènes proviennent d'environ 1800 espèces génomiques différentes. La connaissance de l'association de plusieurs dizaines de gènes et d'opérons proches offre l'avantage de pouvoir cribler par hybridation des gènes susceptibles de coopérer dans une voie métabolique. Les activités enzymatiques sont ensuite mises en évidence après étalement sur des milieux appropriés [39, 40].

\section{Relancer la recherche de nouvelles molécules à haute valeur ajoutée}

Sachant que $70 \%$ des antibiotiques actuels sont produits par moins de $1 \%$ des bactéries cultivables actuellement répertoriées $^{6}$, l'accès à la microflore de différents écosystèmes représente une ressource biologique potentiellement inépuisable. L'utilisation des micro-organismes non cultivables (non cultivés) pour le développement de nouveaux produits à haute valeur ajoutée commence à voir le jour. Ainsi, un nombre croissant de gènes, d'opérons ou d'enzymes d'intérêt industriel ou pharmaceutique ont été identifiés à partir du métagénome de différents environnements tels que le sol, le milieu marin, de symbiontes marins ou de digesteurs anaérobies. Des antibiotiques tels que la turbomycine $A$ et $B$, la violacéine, des anticancéreux tels que les polykétides, des enzymes telles que des polykétide synthases, des lipases/estérases, des $\alpha$-amylases, des chitinases, cellulases, des oxydoréductases, ou encore des nitrilases ont été identifiés [41,42]. L'activité de ces produits a été démontrée par des tests biochimiques ou par des expériences de complémentation génétique. Les micro-organismes hôtes à l'origine de ces gènes et enzymes ne sont pas encore connus. Cette biodiversité microbienne non cultivée, encore inexplorée, intéresse fortement les grands groupes pharmaceutiques, mais aussi des sociétés nord-américaines telles que Diversa, TerraGen Discovery, ou Ariad Pharmaceuticals, et la Société Libragen en France, qui se spécialisent dans ce domaine. Ces micro-organismes non encore cultivés seront d'une importance capitale pour la biotechnologie, la médecine et notre économie future.

\section{Conclusions}

Les méthodes modernes d'étude de la diversité microbienne attirent notre attention sur le fait que la grande diversité du vivant est microbienne. Nous n'avons, en effet, que très peu d'informations sur la plus grande majorité des micro-orga-

\footnotetext{
${ }^{6}$ Les techniques conventionnelles d'exploitation des ressources microbiologiques reposent su le criblage plus ou moins élaboré d'isolats bactériens, permettant de détecter les producteurs de métabolites secondaires tels que les molécules d'intérêt thérapeutique à activité biologique
} antibiotique, anticancéreuse ou anti-inflammatoire. nismes qui colonisent notre planète. Tant que des efforts soutenus ne seront pas déployés pour le développement d'outils et de stratégies permettant leur culture, seuls ou en association avec d'autres micro-organismes, nous serons condamnés à spéculer sur l'importance et le rôle fonctionnel des microorganismes non encore cultivés et sur leur potentiel, aussi bien physiologique que biochimique. $\diamond$

\section{SUMMARY}

Expanding the known diversity and environmental

distribution of cultured and uncultured bacteria

Microorganisms represent the largest component of biodiversity in our biosphere. Traditional methods of bacterial identification depend on their culture on laboratory media and the comparison of their phenotypic characteristics. They include cellular morphology, motility, staining reactions of cell walls, ability to grow on different media and biochemical tests. These methods have many limitations and only a very small fraction of microorganisms have been cultivated. To date, molecular methods based on 16S rRNA sequences and their phylogenetic analysis are widely used for reliable identification, particularly for hard-to-culture microbial pathogens. These so-called «molecular methods » do not require laboratory culture of isolated organisms, and many novel non-described phyla have been detected, improving our view of bacterial diversity. Novel strategies for culturing the « uncultivated » are now under development, which are leading to the complete characterization of these new bacteria. More recently, meta- or ecogenomics, based on the complete sequencing of clones containing cosmids or bacterial artificial chromosomes with inserts, addresses the genetic potential of a sample irrespective of whether the microorganisms can be cultured or not. This has considerably extended our view of microbial diversity at the genomic level and the probability of finding new genes and their products suitable for the biotechnological and pharmaceutical industry. $\nabla$

\section{RÉFÉRENCES}

1. Woese CR. Bacterial evolution. Microbiol Rev 1987; 51 : 221-71.

2. Stackebrandt $\varepsilon$, Goebel BM. Taxonomic note: a place for DNA-DNA reassociation and $16 \mathrm{~S}$ rRNA sequence analysis in the present species definition in bacteriology. Int J Syst Bacteriol 1994; 44 : 846-9.

3. Rappé MS, Giovannoni SJ. The uncultured microbial majority. Annual Rev Microbiol 2003; 57 : 369-94.

4. Dojka MA, Harris JK, Pace NR. Expanding the known diversity and environmental distribution of an uncultured phylogenetic division of bacteria. Appl Environ Microbiol 2000; 4 : 1617-21

5. Dauga C. Evolution of the gyrB gene and the molecular phylogeny of Enterobacteriaceae: a model molecule for molecular systematic studies. Int J Syst Evol Microbiol 2002; 52: 531-47.

6. Kim BJ, Hong SK, Lee KH, et al. Differential identification of Mycobacterium tuberculosis complex and nontuberculous mycobacteria by duplex PCR assay using the RNA polymerase gene (rpoB). J Clin Microbiol 2004; 42: 1308-12.

7. Zeaiter Z, Fournier $P E, 0$ gata $\mathrm{H}$, et al. Phylogenetic classification of Bartonella species by comparing groeL sequences. Int J Syst Evol Microbiol 2002; 52: $165-71$. 
8. Anderson B, Sims K, Regnery R, et al. Detection of Rochalimaea henselae DNA in specimens from cat scratch disease patients by PCR. J Clin Microbiol 1994; 32 : 942-8.

9. Relman DA, Loutit JS, Schmidt TM, et al. The agent of bacillary angiomatosis. An approach to the identification of uncultured pathogens. N Engl J Med 1990; 323 : 1573-80.

10. Relman DA, Schmidt TM, MacDermott, et al. Identification of the uncultured bacillus of Whipple's disease. N Engl J Med 1992; 327: 293-301.

11. La Scola B, Raoult D. Culture of Bartonella quintana and Bartonella henselae from human samples: a 5-year experience (1993 to 1998).

J Clin Microbiol 1999; 37 : 1899-905.

12. Maiwald M, von Herbay A, Fredricks DN, et al. Cultivation of Tropheryma whipplei from cerebrospinal fluid. J Infect Dis 2003; 188: 801-8.

13. Dauga C, Miras I, Grimont PAD. Identification of Bartonella henselae and B. quintana $16 \mathrm{~S}$ rDNA sequences by branch-, genus-, and species-specific amplification. J Med Microbiol 1996; 45 : 192-9.

14. Dauga C, Miras I, Grimont PAD. Strategy for detection and identification of bacteria based on 16S rRNA genes in suspected Whipple's disease. J Med Microbiol 1997; 46: 340-7.

15. Coenye T, Goris J, Spilker T, et al. Characterization on unusual bacteria isolated from respiratory secretions of cystic fibrosis patients and description of Inquilinus limosus gen. nov., sp. nov. J Clin Microbiol 2002; 40: 2062-9

16. Roussée JM, Bermond D, Piémont $Y$, et al. Dialister pneumosintes associated with human brain abscesses. J Clin Microbiol 2002; 40 : 3871-73.

17. Hervio-Heath $D$, Colwell RR, Derrien A, et al. Occurrence of pathogenic vibrios in coastal areas of France. J Appl Microbiol 2002; 92: 1123-35.

18. Pruzzo C, Crippa A, Bertone $S$, et al. Attachment of Vibrio alginolyticus to chitin mediated by chitin-binding proteins. Microbiology 1996; 142: 2181-6.

19. Ballard AL, Fry NK, Chan L, et al. Detection of Legionella pneumophila using a realtime PCR hybridization assay. J Clin Microbiol 2000; 38: 4215-8.

20. Hayden RT, Uhl JR, Qian X, et al. Direct detection of Legionella species from bronchoalveolar lavage and open lung biopsy specimens: comparison of LightCycler $\mathrm{PCR}$, in situ hybridization, direct fluorescence antigen detection, and culture. J Clin Microbiol 2001; 39: 2618-26.

21. Kessler HH, Reinthaler FF, Pschaid A, et al. Rapid detection of Legionella species in bronchoalveolar lavage fluids with the EnviroAmp Legionella PCR amplification and detection kit. J Clin Microbiol 1993; 31: 3325-8.

22. Lindsay DS, Abraham AW, Fallon RJ. Detection of mip gene by PCR for diagnosis of Legionnaires' disease. J Clin Microbiol 1994; 32: 3068-9.

23. Wellinghausen N, Frost C, Marre R. Detection of Legionellae in hospital water samples by quantitative real-time LightCycler PCR. Appl Environ Microbiol 2001; 67: 3985-93.

24. Jonas $D$, Rosenbaum $A$, Weyrich $S$, et al. Enzyme-linked immunoassay for detection of PCR-amplified DNA of Legionellae in bronchoalveolar fluid. J Clin Microbiol 1995; 33: 1247-52.

25. Cloud JL, Carroll KC, Pixton P, et al. Detection of Legionella species in respiratory specimens using PCR with sequencing confirmation. J Clin Microbiol $2000 ; 38: 1709-12$

26. Calvo-Bado LA, Morgan AJ, Sergeant M, et al. Molecular characterization of Legionella populations present within slow sand filters used for fungal plant pathogen suppression in horticultural crops. Appl Environ Microbiol 2003; 69: 533-41.
27. Campese C, Che D. Cluster of legionnaires' disease cases in northern France, the situation on 18 december 2003. Eurosurveillance Weekly 2003; 7 (http://www.eurosurveillance.org/ew/2003/031218.asp).

28. Cora LB, Matthias H, Rolf M, et al. The genus Caedibacter comprises endosymbionts of Paramecium spp related to the Rickettsiales (alpha proteobacteria) and to Francisella tularensis (gamma proteobacteria). Appl Environ Microbiol 2002; 68 : 6043-50.

29. Horn M, Harzenetter MD, Linner T, et al. Members of the CytophagaFlavobacterium-Bacteroides phylum as intracellular bacteria of acanthamoebae: proposal of Candidatus Amoebophilus asiaticus. Environ Microbiol 2001; 3: 440-9.

30. Fritsche TR, Horn M, Wagner M, et al. Phylogenetic diversity among geographically dispersed Chlamydiales endosymbionts recovered from clinical and environmental isolates of Acanthamoeba spp. Appl Environ Microbiol 2000; 66: 2613-9.

31. Amann R, Springer N, Schonhuber W, et al. Obligate intracellular bacterial parasites of Acanthamoebae related to Chlamydia spp. Appl Environ Microbiol 1997; 63: 115-21.

32. Horn M, Wagner M, Muller KD, et al. Neochlamydia hartmannellae gen. nov., sp. nov. (Parachlamydiaceae), an endoparasite of the amoeba Hartmannella vermiformis. Microbiology 2000; 146: 1231-9.

33. Huber R, Burggraf $S$, Mayer T, et al. Isolation of a hyperthermophilic archaeum predicted by in situ RNA analysis. Nature $1995 ; 376$ : 57-8.

34. Rappé MS, Connon SA, Vergin KL, et al. Cultivation of the ubiquitous SAR11 marine bacterioplankton clade. Nature 2002; 418: 630-3.

35. Zoetendal $\varepsilon G$, Plugge CM, Akkermans AD, et al. Victivallis vadensis gen. nov., sp. nov., a sugar-fermenting anaerobe from human faeces. Int J Syst Evol Microbiol 2003; $53: 2111-2$

36. Janssen PH, Yates PS, Grinton BE, et al. Improved culturability of soil bacteria and isolation in pure culture of novel members of the divisions Acidobacteria, Actinobacteria, Proteobacteria, and Verrucomicrobia. Appl Environ Microbiol 2002; 68: 2391-6.

37. Joseph SJ, Hugenholtz $P$, Sangwan $P$, et al. Laboratory cultivation of widespread and previously uncultured soil bacteria. Appl Environ Microbiol 2003; 69: 7210-5.

38. Kaeberlein T, Lewis K, Epstein SS. Isolating «uncultivable» microorganisms in pure culture in a simulated natural environment. Science 2002; 296: 1127-9.

39. Venter JC, Remington K, Heidelberg JF, et al. Environmental genome shotgun sequencing of the Sargasso sea. Science 2004; 304: 66-74.

40. Henne A, Daniel R, Schmitz RA, Gottschalk G. Construction of environmental DNA libraries in Escherichia coli and screening for the presence of genes conferring utilization of 4-hydroxybutyrate. Appl Environ Microbiol 1999; 65 : 3901-7.

41. Daniel R. The soil metagenome: a rich resource for the discovery of novel natural products. Curr Opin Biotechnol 2004; 15 : 199-204.

42. Streit $W R$, Daniel $R$, Jaeger KE. Prospecting for biocatalysts and drugs in the genomes of non-cultured microorganisms. Curr Opin Biotechnol 2004; 15: 285-90.

43. Hugenholtz P, Goebel BM, Pace NR. Impact of culture-independent studies on the emerging phylogenetic view of bacterial diversity. J Bacteriol 1998; 180: 4765-74.
TIRÉS À PART

A. Sghir

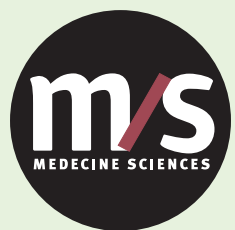

Tarifs d'abonnement M/S - 2005

Abonnez-vous

à Médecine/Sciences
$>$ 1985-2005, depuis 20 ans, grâce à $\mathrm{m} / \mathrm{s}$, vous vivez en direct les progrès des sciences biologiques et médicales
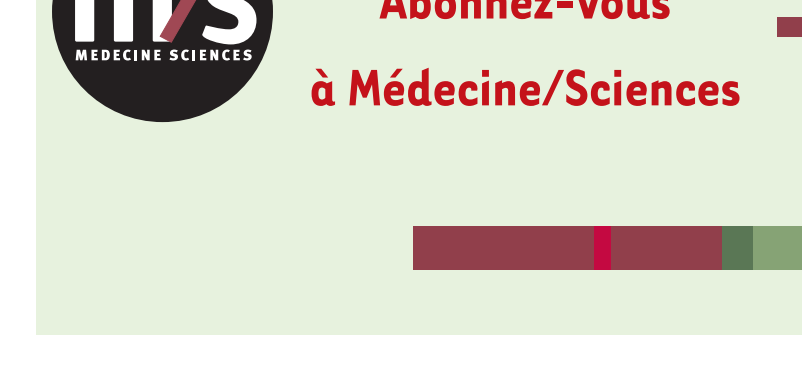

Bulletin d'abonnement

page 252 dans ce numéro de $\mathrm{m} / \mathrm{s}$

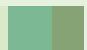

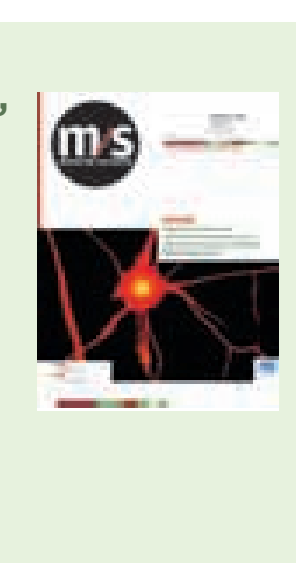

